American Journal of Applied Sciences 9 (10): 1542-1552, 2012

ISSN 1546-9239

(C) 2012 Science Publication

\title{
Close Range Photogrammetry and Neural Network for Facial Recognition
}

\author{
${ }^{1}$ Rami Al-Ruzouq and ${ }^{2}$ Shatha Kadhim \\ ${ }^{1}$ Department of Civil Engineering and Architecture, \\ Faculty of Engineering, University of Bahrain, Manama \\ ${ }^{2}$ Department of Computer Engineering, \\ Faculty of Engineering, Al_Balqa' Applied University, Salt, Jordan
}

\begin{abstract}
Recently, there has been an increasing interest in utilizing imagery in different fields such as archaeology, architecture, mechanical inspection and biometric identifiers where face recognition considered as one of the most important physiological characteristics that is related to the shape and geometry of the faces and used for identification and verification of a person's identity. In this study, close range photogrammetry with overlapping photographs were used to create a three dimensional model of human face where coordinates of selected object points were exatrcted and used to caculate five different geometric quantities that been used as biometric authentication for uniquely recognizing humans. Then, the probabilistic neural networks, with their remarkable ability to derive meaning from complicated or imprecise data, utilize the extracted geometric quantities to find patterns and detect trends that are too complex to be noticed by either humans or other computer techniques. Quantifiable dimensions that based on geometric attributes rather than radiometric characteristics has been successfully extracted using close range photogrammetry. the Probabilistic Neural Network (PNN) as a kind from radial basis network group has been used to specify a geometrics parameters for face recognition where the designed recognition method is not effected by face gesture or color and has lower cost compared with other techniques. This method is reliable and flexible with respect to the level of detail that describe the human surface. Experimental results using real data proved the feasibility and the quality of the suggested approach.
\end{abstract}

Key words: Probabilistic Neural Network (PNN), Computed Tomography (CT), Principal Components Analysis (PCA), Interior Orientation Parameters (IOPs), Single-Lens Reflex (SLR)

\section{INTRODUCTION}

Photogrammetry is concerned with deriving measurements of the size, shape and position of objects from measurements made on photographs. In its simplest form, a pair of overlapping photographs is used to create a three Dimensional (3D) model, which with the use of appropriate instrumentation can yield quantifiable dimensions of the object. Traditionally, these dimensions were represented on maps and plans, either as elevations, facades and/or contours. The use of photogrammetry as a tool to aid in the individual identification and face recognition has a long history and is well established as a measurement science. Recent advances in the science make the techniques much more flexible in their application and present new opportunities in the representation of the human identity features and face recognition. Face recognition is an active research area that has many real-life applications such as identification and verification for terrorists, criminals and missing children, bank and store security, providing an authentication that include the right person with the right privileges and the right access at the right time.

Zappa et al. (2010) presents an innovative facial recognition algorithm where a stereoscopic calibrated system and two images were used to generate 3D model based on 58 homologous points. The triangulation of these points allows the construction of a 3D mask of the face of the individual. This mask is then compared against a database of reference masks. Database showed a false rejection rate identically equal to zero and a false acceptance rate of about 3\%. Moreton and Morley (2011) used Photoanthropometry as a metric based facial image comparison technique. Measurements of the face are taken from an image using predetermined facial landmarks. Measurements are then converted to Proportionality Indices (PIs) and compared to PIs from another facial image. The aim of this study was to

Corresponding Author: Rami Al-Ruzouq, Department of Civil Engineering and Architecture, Faculty of Engineering University of Bahrain, Manama 
Am. J. Applied Sci., 9 (10): 1542-1552, 2012

determine the reliability of photoanthropometric measurements between different images of the same individual taken with different angulations of the camera. The study examined the facial measurements of 25 individuals from high resolution photographs, taken at different horizontal and vertical camera angles in a controlled environment. Results show that the degree of variability in facial measurements of the same individual due to variations in camera angle can be as great as the variability of facial measurements between different individuals. Results suggest that photoanthropometric facial comparison, as it is currently practiced, is unsuitable for elimination purposes. Ayoub et al. (2007) investigates and assists the feasibility of building a virtual human face digitally by superimposing a photo-realistic with 3D soft-tissue surface on bone in the correct relationship and evaluating the registration errors associated with this method. The 3D soft-tissue surface of the face was captured using a fast stereo photogrammetry method and the underlying bone was recorded using a 3D Computed Tomography (CT) scanner. Using the Procrustes registration method, the outer surface of the 3D CT scan and the photorealistic soft-tissue surfaces were merged into a single Virtual Reality.

Bronstein et al. (2004) performs 3D face recognition with isometric signatures where twodimensional pictures of the subjects are shown in the first row. The second row depicts the 3D facial surfaces acquired by a range camera (structured light) and the third row shows the canonical isometric signatures (projections based on geodesic distances). Distances of the subjects from the reference were computed, using a surface matching (first row of numbers) and isometric signature matching (second row of numbers). The small inter-instance distances and the large distance from the control subject when invariant signatures are used. Another approach is based on 2D-facial feature extraction. The sites with statistical pattern matching attractors use the Distance to Feature Space (DF FS) as attractor. The squared Euclidian distance to the space spanned by the principle components for the specific face area, such as: Eigen-mouths and Eigen-eyes. Together, these areas cover the important knowledge of the face. For the eye sites, the iris detector is needed. Some other methods are attracted to edges in order to obtain contours. Khalil (2011) describes a simple approach to measure the displacement of a moving object in two directions simultaneously. The proposed approach is based on static close range photogrammetry with a single camera and the well-known collinearity equations. The proposed approach requires neither multi-camera synchronization nor mutual camera calibration. It requires no prior knowledge of the kinematic and kinetic data of the moving object. The proposed approach was used to evaluate predefined two-dimensional displacements of a moving object. The root mean square values of the differences between the predefined and evaluated displacements in the two directions are 0.11 and $0.02 \mathrm{~mm}$.

Jesorsky et al. (2001) present a shape comparison approach to achieve fast, accurate face detection that is robust to changes in illumination and background. The proposed method is edge-based and works on grayscale still images. The Hausdorff distance is used as a similarity measure between a general face model and possible instances of the object within the image. The study describes an efficient implementation, making this approach suitable for real-time applications. A twostep process that allows both coarse detection and exact localization of faces is presented. System performance has been examined on two large test sets by comparing eye positions estimated by the system against manually set ones with a relative error measure that is independent of both the dimension of the input images and the scale of the faces. The good localization results show that the system is robust against different background conditions and changing illumination. Zhili et al. (2006) present a method for constructing dense correspondences between 3D open surfaces that is sufficiently accurate to permit clinical analysis of $3 \mathrm{D}$ facial morphology. Constructing dense correspondences between $3 \mathrm{D}$ models representing facial surface anatomy is a natural extension of landmark-based methods for analyzing facial shape or shape changes. The method is based on elastic deformation, which deforms a 3D generic model onto the 3D surface of a specific individual. Validation experiments show that, using only five manually placed landmarks, approximately $95 \%$ of triangles on the deformed generic mesh model are within the range of $\pm 0.5 \mathrm{~mm}$ to the corresponding original model. The established dense correspondences have been exploited within a Principal Components Analysis (PCA)-based procedure for comparing the facial morphology of a control group to that of a surgically managed group comprising the patients who have been subject to facial lip repair. It has been noted that some studies had tried to raise the Principle Component Analysis (PCA) face recognition performance by mirror image made out of luminance difference between the left and right against the front face shaded by illumination (Song et al., 2006). Others used PCA to compute distances by combining geometrical features and texture information (Shi et al., 2006; Yambor et al., 2002) or identify the location of the nose in 3D facial data (Jesorsky et al., 2001).

Kumar et al. (2011) presented a face identification algorithm that based on modular localized variation by 
Eigen Subspace technique, in this study The face imagery was partitioned into smaller sub-divisions from a predefined neighborhood and they were ultimately fused to acquire many sets of features. The proposed algorithm argue it ablity to enhanced recognition accuracy for face imagery with illumination, expression and pose variations. Rizon et al. (2006) developed a computational model to identify the human face by applying eigenfaces. The eigenfaces has been applied to extract the basic face of the human face images. Then eigenfaces projecting onto human faces to identify unique features vectors. This features vector was used to identify an unknown face by using the backpropagation neural network that utilized euclidean distance for classification and recognition.

In summary, two major challenge for face recognition were discussed in literature; first, finding the suitable characteristics feature to be used for face recognition, these features could be radiometric wish can changed a long time and raise big quotation about its efficiency while others depend on geometric attributes which is constant along time and would not be affected by radiometric properties such as gesture and colour of the human face. It has to be noted that geometry was built either in $2 \mathrm{D}$ or $3 \mathrm{D}$ where three dimensional would add significant new parameters that participate significantly of recognizable identities of individuals. The second issue is finding an efficient algorithm to derive meaning from complicated or imprecise data and can use the extracted geometric quantities to find patterns and detect trends that are too complex to be noticed by either humans or other computer techniques. In this study geometric properties was adopted rather than radiometric properties where close range photogrammetry were used to extract three demotions features of human face .Several geometrical and mathematical calculations employed to determine the specification and geometric attribute of the human face. Moreover, the Probabilistic Neural Network (PNN) as a kind from radial basis network group has been used to specify geometrics parameters for face recognition process. In this study static probability neural network is used, since it gives high accuracy compared with other types of neural networks. It takes the output of mathematical calculations for each sample and detect if the person is stored in the database.

\section{MATERIALS AND METHODS}

The role of photogrammetry in face recognition: Photogrammetry has been applied to the planning, recording, reconstruction and revitalization of world heritage sites. With the advent of digital photogrammetry and image processing technology, the photogrammetric recording of world heritage sites has rapidly increased.
Working in a digital environment allows flexibility in the choice of computer hardware and software and enables non-photogrammetrists to produce accurate data for recording purposes. Digital object enhancement and 3Dmodeling techniques are also possible and usually give clear presentation of human face. They considerably enhance recognition of construction material, shape and area and their spatial distribution, which is considered as one of the most difficult and time-consuming tasks for architects (Lerma et al., 2000). In summary, photogrammetry offers a rapid and accurate method of acquiring 3D information regarding cultural monuments. Combining the measurements obtained from the photogrammetric record and 3D CAD models offer the means to recreate historic environments. This facilitates the generation of accurate digital records of human faces, while reducing the overall costs.

Before using photogrammetric techniques for face recognition, factors that have an impact on recording accuracy and archiving efficiency have to be discussed: namely, metric characteristics of the camera, imaging resolution and requirements of the bundle adjustment procedure (Chong et al., 2002). Describetion of the mathematical model that incorporates bundle adjustment procedure for accurate estimation of the interior orientation parameters must be discussed. This is a necessary prerequisite for accurate and reliable 3Dcontstruction of the human surface.

Mathmatical model: The basic problem in analytical photogrammetry is to mathematically relate the positions in space of imaged objects to the positions of their image points in the plane of the image and vice versa. The most basic geometric concept utilized in developing solutions to this problem is the collinearity condition which is that the camera station, the image point and the imaged object all lie on a straight line. collinearity equations (mathematical model between image and ground coordinate) appear in the form where the image coordinates of an object are expressed as functions of the Interior Orientation Parameters (IOPs) and the Exterior Orientation Parameters (EOPs) and the space coordinates of the object, (Kubik, 1991). IOPs include image coordinates of the principle point, principle distance and compensations for the deviations from collinearity where EOPs include the ground coordinates of the perspective centre and the elements of the rotation matrix.

The collinearity equations, which define the relationship between image and ground coordinates of a point in the image, are:

$$
\begin{aligned}
& x_{a}=x_{p}-c \frac{r_{11}\left(X_{A}-X_{O}\right)+r_{21}\left(Y_{A}-Y_{O}\right)+r_{31}\left(Z_{A}-Z_{O}\right)}{r_{13}\left(X_{A}-X_{O}\right)+r_{23}\left(Y_{A}-Y_{O}\right)+r_{33}\left(Z_{A}-Z_{O}\right)}+\Delta x \\
& y_{a}=y_{p}-c \frac{r_{12}\left(X_{A}-X_{O}\right)+r_{22}\left(Y_{A}-Y_{O}\right)+r_{32}\left(Z_{A}-Z_{O}\right)}{r_{13}\left(X_{A}-X_{O}\right)+r_{23}\left(Y_{A}-Y_{O}\right)+r_{33}\left(Z_{A}-Z_{O}\right)}+\Delta y
\end{aligned}
$$


Am. J. Applied Sci., 9 (10): 1542-1552, 2012

Where:

$\mathrm{x}_{\mathrm{a}}, \mathrm{y}_{\mathrm{a}}: \quad=$ Image coordinates of the observed image

point a

$\mathrm{X}, \mathrm{Y}, \mathrm{Z}=$ Ground coordinates of object point $\mathrm{A}$

$\mathrm{x}_{\mathrm{p}}, \mathrm{y}_{\mathrm{p}} \quad=$ Image coordinates of the principle point

c: $\quad=$ Camera constant ( principle distance)

$\mathrm{X}_{\mathrm{o}}, \mathrm{Y}_{\mathrm{o}}, \mathrm{Z}_{\mathrm{o}}=$ Ground coordinates of the perspective centre

$\mathrm{r}_{11, \ldots .,} \mathrm{r}_{33}=$ Elements of rotation matrix that are a function of rotation angles $(\omega, \phi, \mathrm{k})$

$\Delta \mathrm{x}, \Delta \mathrm{y}=$ Compensations for the deviations from collinearity

Several methods can be applied to determine the parameters of the orientation of one, two or more photos. The orientation can be processed in steps (relative and absolute orientation) and simultaneous methods (bundle adjustments) that is available in a majority of software packages. For systems based on stereoscopic measurements, the stereo model derives from a relative orientation. In this step, points coordinates are determined in an arbitrary coordinate system of the stereo model. The determination of points in the object coordinate system is done in a second step, known as absolute orientation, by applying a 3D similarity transformation.

For the interior orientation, two sets of parameters have to be considered. The first one contains the geometric parameters of the camera: the principal distance and the coordinates of the principal point. The second set includes the parameters that describe the systematic errors (as distortions or film deformations). At least 5 tie points, that appears in the overlap area of stereo pair, required to extract the 3D model from stereo pair. Connection of an image and its corresponding object space is performed by the (EOPs) of that image. These parameters establish the physical geometry of the camera with respect to ground and describe the position and orientation of that camera at the time of imaging. At least, two full ground control points and one vertical are needed to solve the EOPs based on 3D similarity transformation.

Photogrammetric processing: A calibrated camera is used to capture convergent images for the object to be constructed (Human Face). Tie points are measured in overlapping images and incorporated in a bundle adjustment procedure. For a bundle adjustment, points can be selected in any of the images, these tie points need to be identifiable and visible in other images (conjugate points). Two collinearity equations will be written for each measured conjugate point from each image. For this purpose, an arbitrary datum is chosen and three-dimensional coordinates are determined with respect to that datum. The next paragraph hows in details a case study for the reconstruction of a human surface. The conducted experiment composed of capturing convergent images, measuring of tie points and incorporating these pointes in bundle adjustment procedure for estimation of their 3D-coordinates and finally incorporation of these measurements and establishing quantifiable face recognition quantities, in other words provide some geometrics relations among selected points for improving face recognition system.

Extraction of three dimensional coordinates of based on close range photogrammetry and using multiple images process can be divided into four main steps; first step consist of capturing convergent imagery of human surface that needed to be recognized as part database. Second step consist of photogrammetric that include selection of interest points (point marking) in each image followed by image matching that employed for recognising the conjugate points (same points that appear in different images) this proves is called referencing. Final step, conjugate points would be incorporated in bundle adjustment procedure using collinearity equations to extract the three dimensional coordinates of these points. The last step testing the relations of the face person with the stored database of known faces by using the neural net. The following paragraphs will describe in details all the required stages that include image processing,marking and refrencing and three dimentioanl exatraction of the extarcted points.

Image acquisition: SONY DSC-F707 digital camera is implemented for calibration and stability analysis. The price of this camera is roughly $\$ 700$ USD. It is a Single-Lens Reflex (SLR) camera with a Charged Coupled Device (CCD) has a resolution of $2560 \times 1920$ pixels and $0.004 \mathrm{~mm}$ pixel size. Various Geometric as well as photographic rules that described by has been adopted for image acquisition process (Waldhausl and Ogleby, 1994). These constrains include:

- Preparing control information where long distance between well defined points were measured and used to recover the scale during the bundle adjustment

- Tacking multiple Stereo images all-around the object (human Face) with overlapping ranges from $75-100 \%$. For each object six images has been captured. Three positions of the camera were adopted with almost the same distant between the camera and the object. First position directly in front of the target, the second and third position at angle approximately 45 degree at the right and left direction, Fig. 1. Two images were taken from each position (portrait and landscape)

- Adopting the convergent stereo images case (basedistance-ratio $1: 10$ to $1: 15$ )

- Fixing the inner geometry of the camera, where zoom of the camera and focal length kept constant. The images were taken with one camera 



Fig. 1: Image capturing based on three different positions

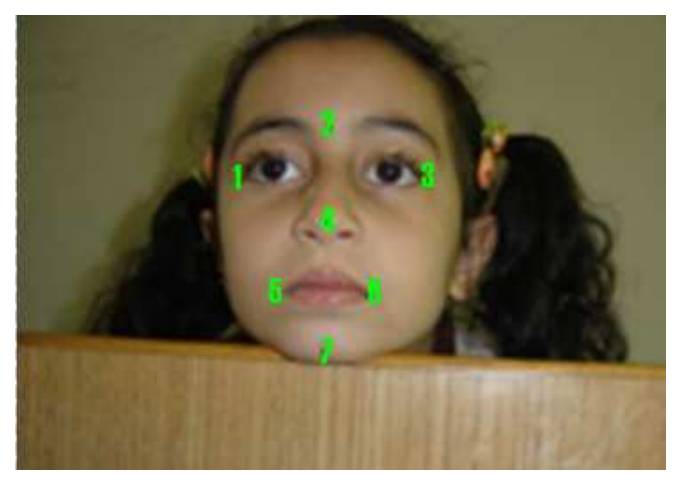

Fig. 2: Interest points of the face
Marking and referencing: This stage includes measuring the interest point (marking) and referencing as well as implementation of bundle adjustment model to extract the three dimensional coordinates of conjugate points. Photo Modeler program as used in this study. The photogrammetric process starts with marking that tend to place and identify the required points on the face picture. Figure 2 shows the interest points on the face target which consist of:

- Eye terminal-points

- Upper middle sections of eye sockets

- Nose-top point

- Mouth terminal-points

- Lower chin point

The relative orientation can be performed by measuring at least five tie points in each stereo-pair. In this work seven points were selected as shown in Fig. 2. it has to be clear that exact points would increase the accuracy and enhance the solutions as the number used to solve unknown parameters in the least squire adjustment increase. It has to mentioned that the 3D coordinates of the mentioned tie points $(1,2,3,4,7)$ would allow for extracting relational attribute of the face rather than radiometric attributes. Recognition that rely on geometric attributes does not affected by the face gesture (sad, happy...), motion of purple, motion of face itself, the colour of skin (colour of skin vary for the same person), existence or absence for sunglass, lenses, or any other face features that may change from time to time.

Marking process is followed by image matching which is an essential aspect of many approaches to problems in computer vision, including object recognition, stereo matching and motion tracking. A well-known approach to image matching consist of identifying the interest points in the images, finding photometric descriptors of the regions surrounding these points and then matching these descriptors across images. This step is called referencing and tends to match each marked point in the reference photo with the equivalent marked point in the other photos considering these equivalent points are related to the same physical object location in space.

\section{RESULTS AND DISCUSSION}

3D coordinate extraction: The extracted conjugate points are then introduced to the colleinrtoty equation and mathematical model to retrieve the three dimensional coordinates of these points. Table 1 contains summary information for the face point locations in space (3D point. The information appears in Table 1 contains Identifier (Id) in the first column which gives a unique number to each selection point. 
Column two shows the image identifier where a specific point appear. The other columns that display the three dimensional coordinates $(\mathrm{X}, \mathrm{Y}, \mathrm{Z})$ value for each interest point in the space.

Geometrical rules: After determining the 3D model for face points, there is a need for extracting rules and relations among them as a required specification for each 3D model. So, fixed mathematical relations among these points can be obtained, such as: distances, areas, volumes, angles and circumferences.

Distance: Calculate the length between two vertices, as shown in Fig. 3. This Figure shows various distance measurements such as between eyes terminators. This indicates that when eyes become wider the distance value increases.

Equation 1 can be used to caculate the distance can between two points. If Point 1 locates at $\left(\mathrm{x}_{1}, \mathrm{y}_{1}, \mathrm{z}_{1}\right)$, Point 2 locates at $\left(\mathrm{x}_{2}, \mathrm{y}_{2}, \mathrm{z}_{2}\right)$, then:

$\mathrm{P}=\left(\mathrm{x}_{1}-\mathrm{x}_{2}, \mathrm{y}_{1}-\mathrm{y}_{2}, \mathrm{z}_{1}-\mathrm{z}_{2}\right)$

$\mathrm{P}=\left(\mathrm{P}_{1}, \mathrm{P}_{2}, \mathrm{P}_{3}\right)$

Distance $=$ Length of $\mathrm{P}(\|\mathrm{P}\|)$ : Eq. 1

$$
\|\mathrm{P}\|=\sqrt{\left(\mathrm{P}^{2}+\mathrm{P} 2^{2}+\mathrm{P} 3\right.}
$$

Area: Calculate the area of triangular that constructed from three vertices as shown in Fig. 4. Figure 4 illustrates various the areas configurations such as the first right image that covers eyes terminators and topnose vertices. This indicates if eyes become wider or nose-high increases then the area value will increase.

If $P_{1}\left(X_{2}-X_{1}, Y_{2}-Y_{1}, Z_{2}-Z_{1}\right)$ locates at one side of triangular and $\mathrm{P}_{2}\left(\mathrm{X}_{2^{-}} \mathrm{X}_{1}, \mathrm{Y}_{2^{-}} \mathrm{Y}_{1}, \mathrm{Z}_{2^{-}} \mathrm{Z}_{1}\right)$ locates on another side at angle with $\mathrm{P} 1$, then equation 2 can be used to caculate the area of the trainagle that include two vectors $\mathrm{P}_{1}$ and $\mathrm{P}_{2}$ Eq. 2:

Area $=\left\|\mathrm{P}_{1} \times \mathrm{P}_{2}\right\| / 2$

Volume: In this geometric parameter, the sphere or pyramid that human face occupies from all Triangular (T) in a space were calculated. Figure 5 illustrates the volume of pyramid that constructed from the main calculated triangular.

Table 1: Three dimensional Space coordinates of interest point

\begin{tabular}{lllll}
\hline Id & Photos & $\mathrm{X}(\mathrm{m})$ & $\mathrm{Y}(\mathrm{m})$ & $\mathrm{Z}(\mathrm{m})$ \\
\hline 1 & $1,2,3,6$ & 0.055992 & 0.012875 & 0.832833 \\
2 & $1,2,3,4 \ldots$ & 0.036545 & 0.058214 & 0.748546 \\
3 & $1,2,3,5 \ldots$ & 0,170790 & 0.011660 & 0.783148 \\
4 & $1,2,3,4 \ldots$ & 0.036512 & 0.056074 & 0.710555 \\
5 & $1,2,3,4 \ldots$ & 0.019907 & 0.124131 & 0.805406 \\
6 & $1,2,3,4 \ldots$ & 0.125905 & 0.121692 & 0.772085 \\
7 & $1,2,3,4 \ldots$ & 0.048518 & 0.186594 & 0.756511 \\
\hline
\end{tabular}
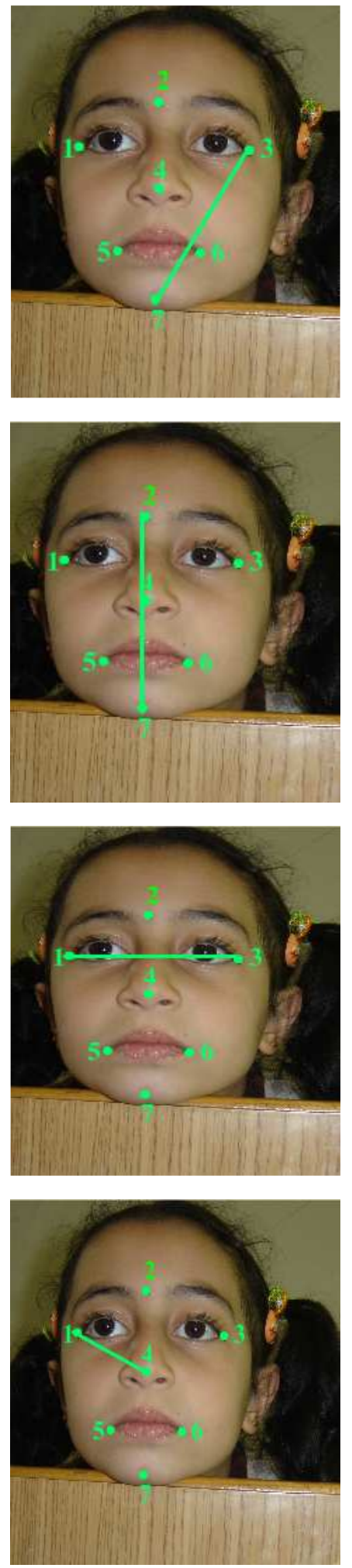

Fig. 3: Three dimensional distant measurements among interest points 

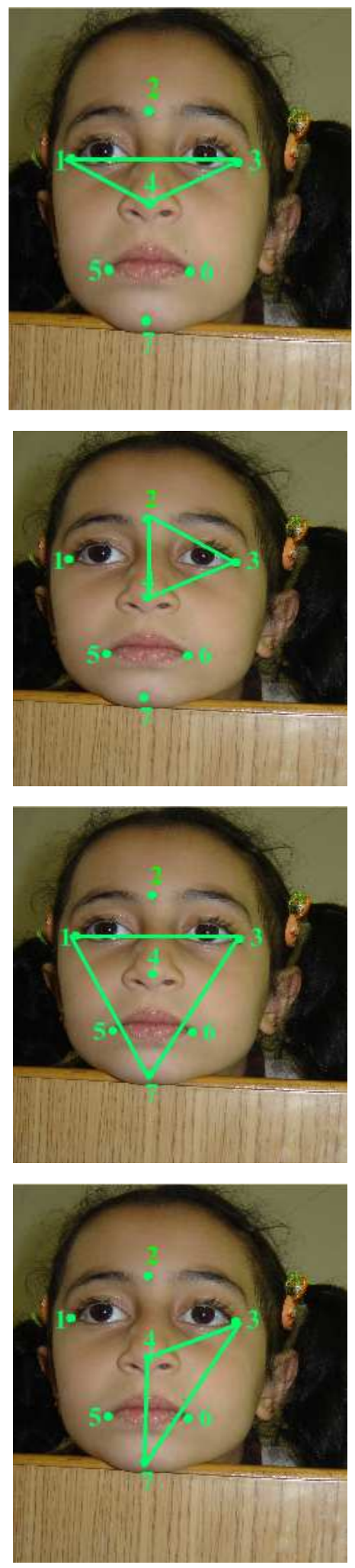

Fig. 4: Area measurements among interest points

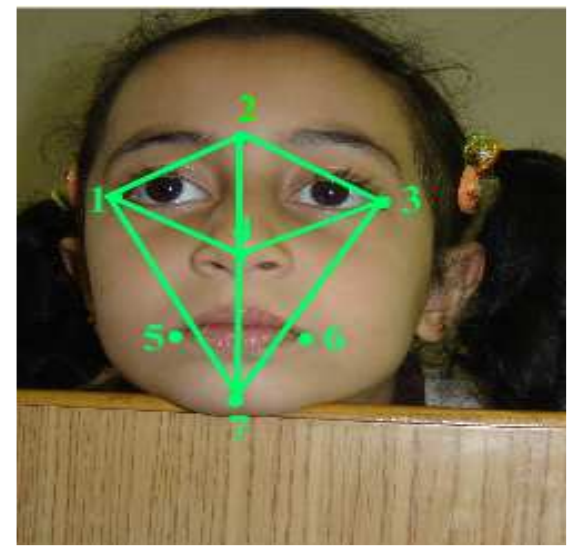

Fig. 5: Volume of pyramid that constructed from the main calculated triangular

This indicates if the human face becomes fully or nose-member is higher, then the volume value will increase and can be calculated using Eq. 3:

Volume $=$ area of $\mathrm{T} *$ high:

$=\left\|\mathrm{P}_{1} \times \mathrm{P}_{2}\right\| / 2 * \mathrm{Z}$-axis value

Angle : Calculate the degree of deflection between two distances or sides as shown in Fig 6. For example, Fig. $6 \mathrm{c}$ shows the measurement of the deflection degree between eye-terminator and chin-point. This indicates if human face closer to cylinder than circular then the value of angle will increase.

If $P_{1}\left(X_{2}-X_{1}, Y_{2}-Y_{1}, Z_{2}-Z_{1}\right)$ vector in space and $P_{2}$ $\left(X_{2^{-}} X_{1}, Y_{2^{-}} Y_{1}, Z_{2^{-}} Z_{1}\right)$ another vector at angle with $P_{1}$. Then equation 4 can be used to caculate the this angle as follows Eq. 4:

Angle $=\sin ^{-1}\left(\left\|\mathrm{P}_{1} \mathrm{x} \mathrm{P}_{2}\right\| /\left(\left\|\mathrm{P}_{1}\right\| *\left\|\mathrm{P}_{2}\right\|\right)\right)$

Circumference: Calculate the summation of distances that performs a closed shape or polygon, as shown in Fig. 7. For example, Fig. 7c illustrates the circumference which covers approximately humanface. This indicates if the human face becomes more cylinders then the circumference value will increase.

If $\mathrm{P}_{1}\left(\mathrm{X}_{2^{-}} \mathrm{X}_{1}, \mathrm{Y}_{2^{-}} \mathrm{Y}_{1}, \mathrm{Z}_{2^{-}} \mathrm{Z}_{1}\right)$ side in a closed polygon, $P_{2}\left(X_{2^{-}} X_{1}, Y_{2^{-}} Y_{1}, Z_{2^{-}} Z_{1}\right.$ another side, $P n$ $\left(X_{2}-X_{1}, Y_{2}-Y_{1}, Z_{2}-Z_{1}\right)$ then the circumference can be cacultaed using Eq. 5 as follows:

Circumference $=\sum$ polygon sides:

$=\left\|\mathrm{P}_{1}\right\|+\left\|\mathrm{P}_{2}\right\|+\ldots+\left\|\mathrm{P}_{\mathrm{n}}\right\|$ 


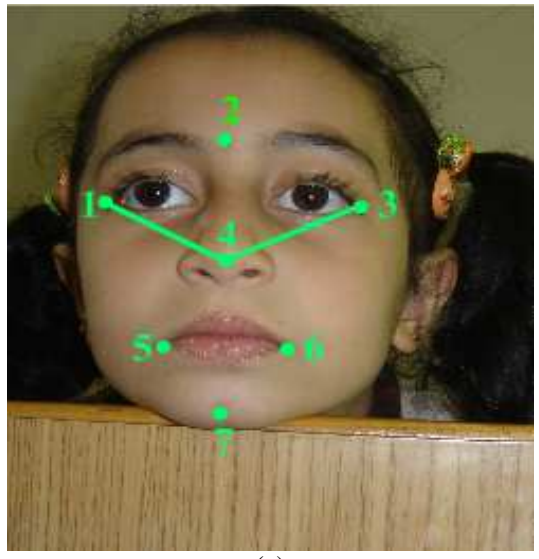

(a)

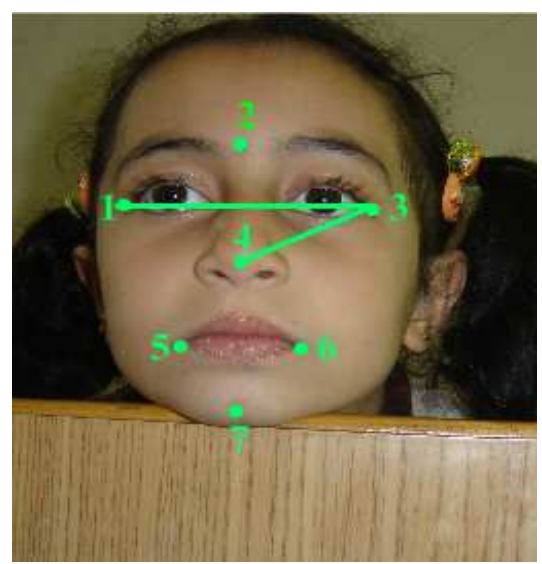

(b)

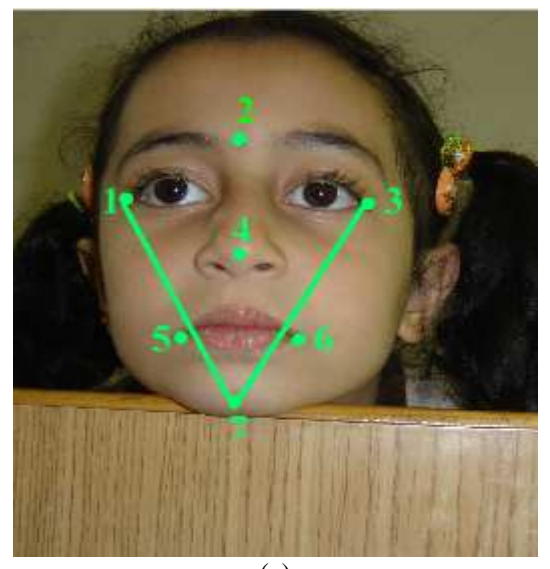

(c)

Fig. 6: Three dimensional Measurements according to Angles

Circumferences in Fig. 7a is ignored, because points on mouth terminals not fixed and affected with face gesture (happy, laugh, sad).



(a)

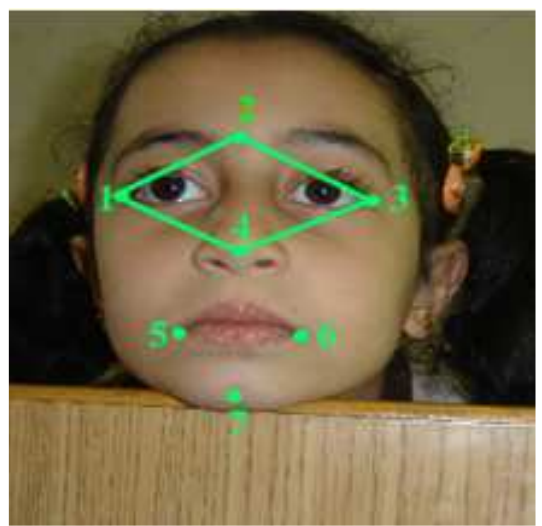

(b)

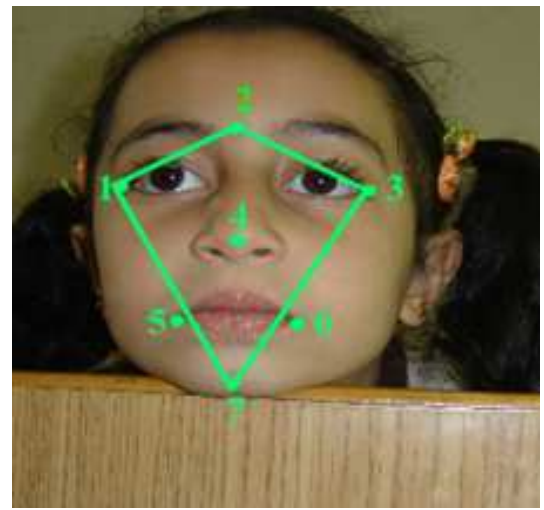

(c)

Fig. 7: Human-face circumference for closed shape or polygon.

Pattern extraction using neural network: The collection of the previous measured face parameters (distances, areas, volumes, angles and circumferences) for each person considered as unique identification between people. Geometrics results enrolled as a database and a new person can compared to the database for authentication using a neural network. Neural networks, with their remarkable ability to derive 
meaning from complicated or imprecise data, can be used to extract patterns and detect trends that are too complex to be noticed by either humans or other computer techniques (Aleksander and Morton, 1995). In this study, the Probabilistic Neural Network (PNN) as a kind from radial basis network group has been used. Such a neural network for classification problems gives accurate output and deals with analog numbers (Davalo et al., 1991).

Figure 8 illustrates the architecture of the PNN; it has two layers; Radial Basis Layer and Competitive Layer. The neural net input represents the geomantic parameters for the new person. The first layer computes distances from the input vector to the training database and produces a vector whose elements indicate how close the input is to a training database. The second layer sums these contributions for each class of inputs to produce as its net output a vector of probabilities. Finally, a compete transfer function on the output of the second layer picks the maximum of these probabilities and the final output 0 if the input person not enrolled in the database, else output give the index of the registered person as identification.

PNN radial basis layer: Radial Basis Layer formalizes the target of all Radial Basis Neural network. This layer takes the input vector and measures the convergence between the input vector and the stored weight vectors. In this layer the Radbas transfer function is used to limit all the distance from unlimited value to a fraction value (0-1) as bellow:

$\mathrm{A}=\operatorname{radbas}(\|\mathrm{W}-\mathrm{P}\| * \mathrm{~b})$

Where:

$\mathrm{P}=\mathrm{A}$ vector of size $\mathrm{R}$

$\mathrm{R}=$ Number of test-person parameters (facedistances, face-volume, ).

$\mathrm{W}=$ Matrix of size $(\mathrm{S} \times \mathrm{R})$

$\mathrm{S}=$ The number of enrolled-samples
$\mathrm{B}=$ The bias which equals to 0

The bias used to adjust the sensitivity of the radbas neuron. The output for Radial Basis Layer is $\mathrm{S} \times 1$ distance matrix which represents the converge degree between the enrolled-samples and the testsample. This converge value has a narrow domain (01), 1 means enrolled-sample and test-sample is identical and vice versa.

PNN competitive layer: Competitive Layer employs compete function for classifying the input vector (testperson) to the identical or approximately identical someone enrolled-sample (approximately identical means the closer person with small error percentage value refers to the percentage of distance between the test and enrolled-sample). The stages and procedures for Competitive Layer are described below:

- Create Target Weight matrix of size $\mathrm{S} \times \mathrm{S}$ with logical ones values; $S$ rows equal to the number of enrolled-samples and $S$ columns equal the number of possible input vectors that can consider as identical to the enrolled-samples

- Produce $S \times 1$ indicator matrix by multiplying weight matrix by the distance matrix. Number of columns in the indicator matrix equal to the number of input vector (one input vector for testperson enter the neural so as especial case the number of columns is one), every cell in the indicator matrix row is a pointer to measuring the percentage closer between each enrolled-sample and the test-person parameters. Largest value in the indicator vector represents the closer enrolledsample for the test-person

- Calculate the output for the neural network by using || compete || transfer function for indicator matrix. The output equal to the index of the matched enrolled-sample

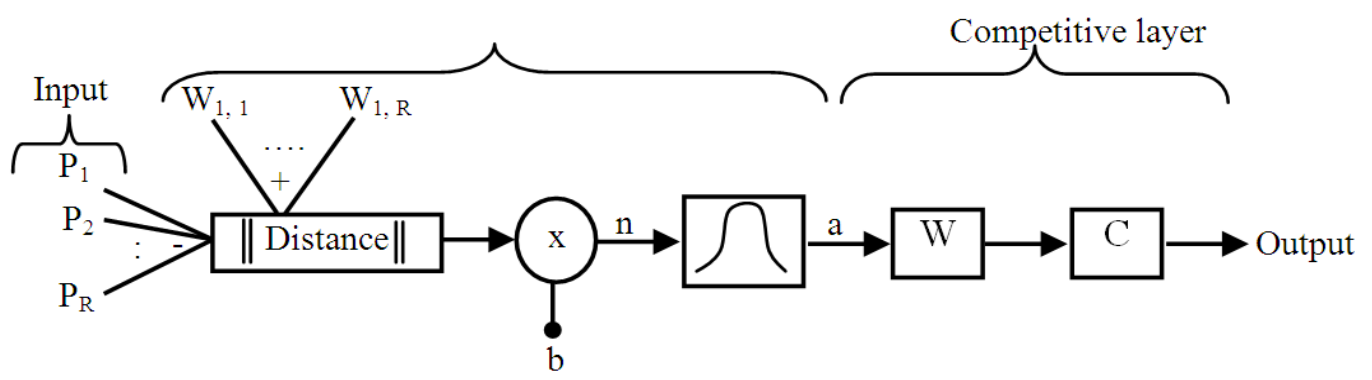

Fig. 8: Architecture of the probabilistic neural network 


\section{CONCLUSION}

In this study, we have presented an operational approach for the reconstruction of Human surface based on photogrammetric techniques. This method relies on bundle adjustment model and collinearity equations. The approach requires accurately measured 3-D points where Marking, matching and three coordinate extraction has been extracted and used to draw the geometric relations of human surface. This method is reliable and flexible with respect to the level of detail that describe the human surface. Future photogrammetric steps may consider the following points:

- Camera calibration is prerequisite to insure interior orientation parameters of the mathematical model

- Automatic matching technique is necessary for reliable face recognition

- More sophisticated geometric relations between the tie points can be considered in order to obtain more accurate face recognition

In this study the Probabilistic Neural Network (PNN) was implemented with 30 samples of known human-faces. The result that obtained from the PNN can be summarized as follows:

- If the test-person matches with any of the enrolledsamples, neural will output the number or index of the matching enrolled-sample

- If the test-person does not match with any of the enrolled-samples, neural will output 0

- Face recognition system very sensitive, because the neural output must determine an identical person. therefore, compete function was enhanced such that the output will be pointed to the largest closer value in the distance matrix which must have similarity greater than $95 \%$ where the accepted error tolerance (1-3 pixels) was obtained. Future work may consider the following points

- The probabilistic neural network deals with multiple entry persons and gets the recognition results for all of them at the same time with low delay

- As a future work, Fuzzy logic can be used to deal with face parameters for recognition

Finally, it has to be mentioned that face recognition using both biometric and geometrics techniques is more accurate compared with other biometric face recognition. The designed 3D face recognition system has lower cost compared with others. Techniques. Moreover the designed recognition method do not effected by face gesture (sad, happy ...), motion of purple, motion of face itself (location between the selected face's points), the colour of the skin and existence or absence for lenses or any temporary features that may added to the human face.

\section{REFERENCES}

Aleksander, I. and H. Morton, 1995. An Introduction to Neural Computing. 2nd Edn. Intl Thomson Computer Pr (T), ISBN-10: 1850321671, pp: 288.

Ayoub, A.F., Y. Xiao, B. Khambay, J. Siebert and D. Hadley, 2007. Towards building a photo-realistic virtual human face for craniomaxillofacial diagnosis and treatment planning. Int. J. Oral Maxillofac Surg., 36: 423-428. DOI: 10.1016/j.ijom.2007.02.003

Bronstein, A.M., M.M. Bronstein, E. Gordon and R. Kimmel, 2004. Fusion of $2 \mathrm{~d}$ and $3 \mathrm{~d}$ data in threedimensional face recognition. Israel Institute of Technology, Israel.

Chong. A., B. McFadgen, Z. Majid, H. McKinlay and S. Luther et al., 2002. Digital architectural photogrammetric recording of historical buildings and monuments. New Zeland Surveyor, 293: 2528.

Davalo, E., P. Naim and A. Rawsthorne, 1991. Neural networks. MacMillan Education.

Jesorsky, O., K. Kirchberg and R. Frischholz, 2001. Robust face detection using the Hausdorff distance. Proceedings of the 3rd International Conference on Audio-and Video-Based Biometric Person Authentication, (AVBBPA' 01), Springer-Verlag London, UK, pp: 90-95.

Khalil, A.M., 2011. Two-dimensional displacement measurement using static close range photogrammetry and a single fixed camera. Alexandria Eng. J., 50: 219-227. DOI: 10.1016/j.aej.2011.07.003

Kubik, K., 1991. Relative and absolute orientation based on linear features. ISPRS J. Photogrammetry Remote Sensing, 46: 199-204. DOI: 10.1016/09242716(91)90053-X

Kumar, N., R. Swami, M. Karuppiah N. Islam and K. Thuckalay et al., 2011 An improved face recognition technique based on modular LPCA approach. J. Comput. Sci., 7: 1900-1907. DOI: 10.3844/jcssp.2011.1900.1907

Lerma, J.L., L.R. Angel and F. Buchon, 2000. Application of spectral and textural classifications to recognize materials and damages on historic building facades. Int. Arch. Photogrammetry Remote Sens., 33: 480-484. 
Moreton, R. and J. Morley, 2011. Investigation into the use of photoanthropometry in facial image comparison. Forensic Sci. Int., 212: 231-237. DOI: 10.1016/j.forsciint.2011.06.023

Rizon, M., M. Hashim, P. Saad, S. Yaacob and M. Mamat et al., 2006. Face recognition using eigenfaces and neural networks. Am. J. Applied Sci., $\quad 2$ : $1872-1875$. DOI: 10.3844/ajassp.2006.1872.1875

Shi, J., A. Samal and D. Marx, 2006. How effective are landmarks and their geometry for face recognition. J. Comput. Vis. Image Understand., 102: 117-133. DOI: 10.1016/j.cviu.2005.10.002

Song, Y.J., Y.G. Kim, U.D. Chang and H.B. Kwon, 2006. Rapid and brief communication: Face recognition robust to left/right shadows; facial symmetry. J. Patt. Recogn., 39: 1542-1545. DOI: 10.1016/j.patcog.2006.02.018
Waldhausl, P. and C. Ogleby, 1994. Close range techniques and machine vision. Proceedings of the ISPRS Symposium Commission V in Melbourne, (CVM' 94), Australia, pp: 1-4.

Yambor, W.S., B.A. Draper and J.R. Beveridge, 2002. Analyzing PCA-Based Face Recognition Algorithms: Eigenvector Selection and Distance measures. Empirical Evaluation Methods Incomputer Vision, H. Christensen and J. Phillips, Eds., Singapore, World Scientific Press, pp: 1-14.

Zappa, E., P. Mazzolenia and Y. Haib, 2010. Stereoscopy based 3D face recognition system. International conference on computational science. ICCS Procedia Comput. Sci., 1: 2521-2528. DOI: 10.1016/j.procs.2010.04.285

Zhili, M., J. Xiangyang, J.P. Siebert, W.P. Cockshott and A.F. Ayoub, 2006. Constructing dense correspondences for the analysis of 3D facial morphology. Patt. Recogn. Lett., 27: 597-608. DOI: 10.1016/j.patrec.2005.09.025 